\title{
Synthesis of Isatin Derivative on the Short Multiwalled Carbon Nanotubes and Their Effect on the MKN-45 and SW742 Cancer Cells
}

\author{
Hasan Tahermansouri and Farshid Chitgar \\ Department of Chemistry, Ayatollah Amoli branch, Islamic Azad University, P.O. Box 678, Amol, Iran \\ Correspondence should be addressed to Hasan Tahermansouri; h.tahermansuri@iauamol.ac.ir
}

Received 9 May 2013; Revised 8 July 2013; Accepted 11 July 2013

Academic Editor: Dario Pasini

Copyright (c) $2013 \mathrm{H}$. Tahermansouri and F. Chitgar. This is an open access article distributed under the Creative Commons Attribution License, which permits unrestricted use, distribution, and reproduction in any medium, provided the original work is properly cited.

\begin{abstract}
The carboxylated short multiwalled carbon nanotubes (Sh-MWCNT-COOH) were first modified by isatin derivative to form Sh-MWCNT-Amide and then by hydrazine to produce Sh-MWCNT-Spiro. All products were characterized by the Fourier transform infrared spectroscopy, Raman spectroscopy, scanning electron microscope, elemental analysis, thermogravimetric analysis, derivative thermogravimetric, solubility test, and cellular investigations. This functionalization has been chosen due to active sites of $\mathrm{OH}$ and carbonyl groups in Sh-MWCNT-Amide which might be used as functional materials in the future. Toxicity of these samples was evaluated with human gastric and colon cancer cells, and killed cell numbers were measured by reduction of living cells with 3-(4,5-dimethyl-2-thiazolyl)-2,5-diphenyltetrazolium bromide. Cellular results showed high toxicity of Sh-MWCNTAmide in all samples investigated.
\end{abstract}

\section{Introduction}

One of the representative paradigms among nanostructured carbon materials, carbon nanotubes (CNTs), has attracted great attention because of remarkable properties in the fields of molecular electronics, sensors, composite materials, and cancer therapy [1-3]. The possibility of functionalization of CNTs, together with their use as nanocontainers for chemical reactions, is in close analogy with recently developed, assembled, nanotubular carbon-based structures $[4,5]$. The potential use of MWCNTs to treat several types of cancer, with minimal or no toxic effects on normal cells, was reported in two papers [6, 7]. Also, biomedical applications of CNTs are investigated actively due to their useful combination of size and physicochemical properties $[8,9]$. Recently, the functionalization schemes enabling the implementation of new functions have developed [10]. Functionalized CNTs were shown to cross cell membrane and deliver attached cargos into cells, in which these properties could be exploited in applications such as gene delivery system, therapeutic siRNA silencing, and molecular imaging $[11,12]$. On the other hand, covalent functionalization is one interesting and efficient way to modify the properties of CNTs such as their solubility. The pristine CNTs are poorly dispersible in both aqueous and organic solvents because their processability is tremendously difficult, but functionalized CNTs can improve the solubility and processability. Thus, the extensive researches have been focused on the functionalization of CNTs which can be cited as cycloaddition [13], oxidation [14], hydrogenation [15], and amidation [16-21]. Also, review articles have been dedicated to this topic $[22,23]$. In this current study, we have investigated the attachment of isatin derivative, 3-(2-amino-1-methyl-4-oxo-4,5-dihydro1H-imidazol-5-yl)-3-hydroxyindolin-2-one (Sh-MWCNTAmide) on the Sh-MWCNT-COOH to produce 5-amino-4methyl-2,4-dihydro-1H-spiro[imidazo[4,5-c]pyrazole-3, $3^{\prime}$ indolin]-2' -one (Sh-MWCNT-Spiro) by the reaction with hydrazine.

The wider range of increasing nanotechnology applications for CNTs will almost certainly result in the increased potential for both human and environmental exposures to this nanomaterial. Therefore, it is imperative to thoroughly 
investigate the toxicity of CNTs. In fact, regarding the potential toxicity of CNTs, there is limited information available in the literatures [24-27]. With this background and by considering potential anticancer agent of this compound [28], isatin derivative, we have investigated the effect of modified MWCNTs on the cancer cells. The toxicity investigations of modified Sh-MWCNTs were carried out on the cancer cells of human gastric and colon. Cellular results showed a high toxicity of Sh-MWCNT-Amide on cancer cells as compared to other samples.

\section{Experimental}

All reagents and solvents (hydrazine hydrate, dicyclohexylcarbodiimide (DCC), tetrahydrofuran (THF), dimethylsulfoxide (DMSO), creatinine, and isatin) from Merck Chemical Inc. and Short-MWCNTs-COOH (95\% purity, OD: $10-20 \mathrm{~nm}$, length: $0.5-2 \mu \mathrm{m}$, Neutrino Co., Ltd.) were purchased and used as received. 3-(2-Amino-1-methyl-4oxo-4,5-dihydro-1H-imidazol-5-yl)-3-hydroxyindolin-2-one is prepared from the reaction of creatinine with isatin [28]. FT-IR spectrum was recorded using $\mathrm{KBr}$ tablets on a Thermo Nicolet Nexus 870 FTIR Spectrometer. Raman spectra, recorded on Almega Thermo Nicolet Dispersive Raman Spectrometer (532 nm of a Nd: YLF laser). FE-SEM was used to study the morphology of the MWCNTs. FE-SEM measurement was carried out on the Hitachi S4160 (Cold Field Emission) Electron Microscope. Elemental analyses of C, H, N were performed with a 2400 Series II from PerkinElmer Co., USA. The samples were investigated by thermogravimetric analysis (NETZSCH TG 209 F1 Iris) in the $\mathrm{N}_{2}\left(10^{\circ} \mathrm{C} / \mathrm{min}\right)$.

2.1. Preparation of Sh-MWCNT-Amide. $150 \mathrm{mg}$ of the ShMWCNT-COOH was thoroughly dispersed in $30 \mathrm{~mL}$ of DMSO by sonication. To this dispersion of the Sh-MWCNT$\mathrm{COOH}, 10 \mathrm{~mL}$ DMSO solution dissolving with $350 \mathrm{mg}$ of isatin derivative was added. Then, $10 \mathrm{~mL}$ of THF solution of an excess amount of dicyclohexylcarbodiimide (DCC) was added dropwise to this solution and stirred at room temperature for $96 \mathrm{~h}$. Then, the mixture was filtered and washed thoroughly with DMSO, ethyl alcohol, and THF. Subsequently, the black solid was dried at room temperature for $8 \mathrm{~h}$ under vacuum condition.

2.2. Preparation of Sh-MWCNT-Spiro. $70 \mathrm{mg}$ Sh-MWCNTAmide was sonicated in $10 \mathrm{~mL}$ DMSO for 15 minutes giving out a homogeneous suspension. Then, $20 \mathrm{~mL}$ hydrazine was added to the reaction mixture and it was stirred at $80^{\circ} \mathrm{C}$ for $96 \mathrm{~h}$. After cooling to room temperature, the reaction mixture was separated by centrifugation and washed thoroughly with ethyl alcohol and THF. Thus, the obtained solids were dried by vacuum for $6 \mathrm{~h}$.

\subsection{Cellular Study}

2.3.1. Materials. The following substances were obtained from the sources as indicated. Cell culture medium
(RPMI1640), fetal calf serum, $0.25 \%$ trypsin with $1 \mathrm{mM}$ of ethylene diamine tetraacetate (EDTA), streptomycin sulfate, and penicillin G sodium, all from GIBCO (Grand Island, NY, USA), were obtained. Human gastric cancer cell lines (MKN45) (NCBI number C615) and human colon cancer cell lines (SW742) were provided by the Iranian Pastor Institute Cell Bank (Tehran, Iran).

2.3.2. Cell Culture. MKN-45 or SW742 was cultured in RPMI1640 supplemented with 10\% fetal bovine serum and $1 \%$ L-glutamine, penicillin, and streptomycin. For these experiments, cells were seeded at a density of $1 \times 10^{5}$ cells $/ \mathrm{mL}$ in 96-well plates. At least three time points are depicted for each assay.

2.3.3. Cell Proliferation. The MTT assay (Sigma) was used to evaluate the proliferative activity. Cells grown in 96well plates were exposed to modified Sh-MWCNTs. Exposure to $50 \mu \mathrm{L}$ RPMI1640 was used as a negative control and $50 \mu \mathrm{L}$ dimethyl sulfoxide as a positive control. After $48 \mathrm{~h}, 20 \mu \mathrm{L}$ of 3-(4,5-dimethyl-2-thiazolyl)-2,5-diphenyl-2Htetrazolium bromide (MTT) solution at $0.5 \mathrm{mg} / \mathrm{mL}$ in Hank's balanced salt solution was added to each well and incubated for $4 \mathrm{~h}$. Afterward, $150 \mu \mathrm{L}$ of the MTT solution (10\% Triton $\mathrm{X}-100$ in $0.1 \mathrm{~N} \mathrm{HCl}$ in anhydrous isopropanol) was added to each well. The resulting formazan crystals were solubilized in acidic isopropanol and quantified by measuring absorbance at $570 \mathrm{~nm}$ with microplate reader (Rayto RT-6100). Data were calibrated to the appropriate calibration curve, as stated in Sigma protocols.

2.3.4. Statistics. All data were collected using Excel. Graphing and statistics were performed with SPSS 11.5. Continuous variables were presented as mean $\pm \mathrm{SD}(x \pm s)$. Data were analyzed using one-way ANOVA to determine the difference among groups. Relative growth rate (RGR) of cells was calculated using the following formula: RGR $=\mathrm{OD}$ of each group/OD of natural group $\times 100 \%$. To determine the cytotoxic response of the Sh-MWCNTs to cells in culture, 6graded toxicity and other analytic methods were used.

\section{Results and Discussion}

Figure 1 illustrates the procedure for functionalization of ShMWCNT-COOH by isatin derivative and hydrazine. This functionalization has been chosen due to active sites of $\mathrm{OH}$ and carbonyl groups in Sh-MWCNT-Amide which might be used as functional materials in the future. The functionalized CNTs were characterized by elemental analysis, Fourier transform infrared spectroscopy (FT-IR), Raman, scanning electron microscope (SEM), thermo gravimetric analysis (TGA), derivative thermogravimetric (DTG), and solubility test.

Elemental analyses of modified Sh-MWCNTs are shown in Table 1. Apart from the carbon values, the changes of the atomic percentages $\mathrm{H}(1.09 \%)$ and $\mathrm{N}(2.86 \%)$ in Sh-MWCNTAmide and $\mathrm{H}(1.12 \%)$ and $\mathrm{N}(3.97 \%)$ in Sh-MWCNT-Spiro (as compared to that in Sh-MWCNT-COOH) indicate that 


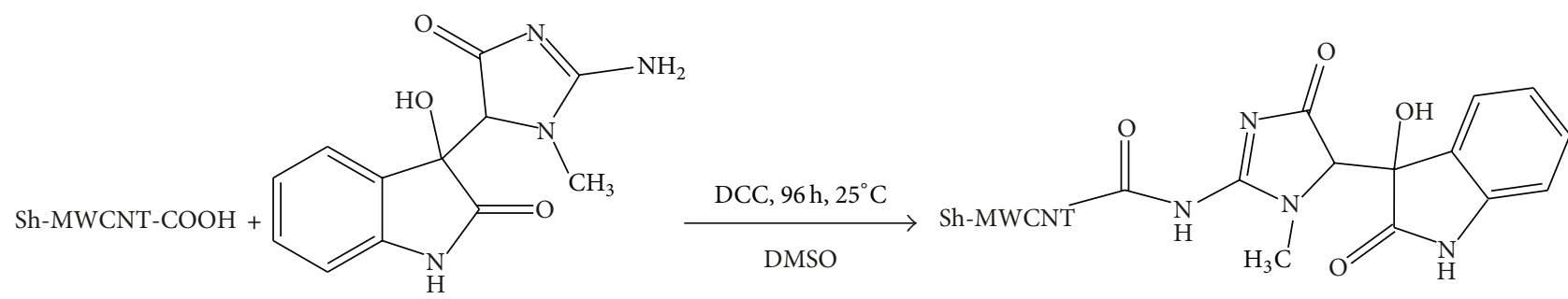

Isatin derivative<smiles>Cn1c(NC(=O)[N-]CCCCCN)nc2c1C1(NN2)C(=O)Nc2ccccc21</smiles>

Sh-MWCNT-Spiro

FIGURE 1: Synthesis route of modified Sh-MWCNT-COOH.

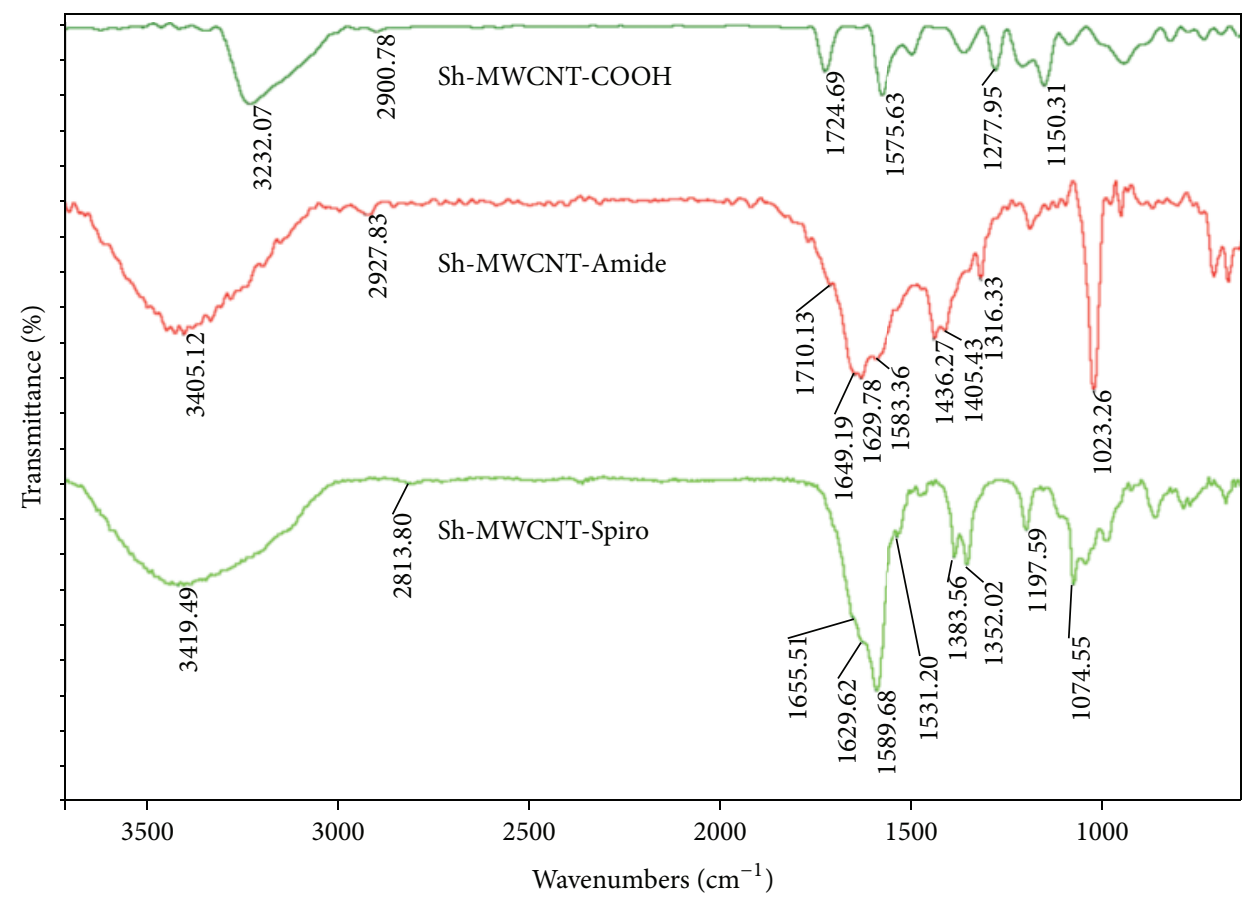

FIGURE 2: FT-IR spectra (after baseline correction) of modified-MWCNTs.

TABLE 1: Elemental analysis of modified Sh-MWCNTs.

\begin{tabular}{lccc}
\hline Modified-MWCNTs & $\% \mathrm{C}$ & $\% \mathrm{H}$ & $\% \mathrm{~N}$ \\
\hline Sh-MWCNT-COOH & 96.34 & 0.72 & 0.00 \\
Sh-MWCNT-Amide & 91.93 & 1.09 & 2.86 \\
Sh-MWCNT-Spiro & 89.11 & 1.12 & 3.97 \\
\hline
\end{tabular}

Sh-MWCNT-COOH is functionalized. On the other hand, the increase of the $\mathrm{N}$ percentage in Sh-MWCNT-Spiro compared to Sh-MWCNT-COOH and Sh-MWCNT-Amide confirms the formation of Spiro compound on the MWCNTs. Based on these data, coupled with the assumption that atomic percentages of nitrogen and hydrogen originate from the employed isatin derivative and hydrazine, we confirmed the functionalization of Sh-MWCNT-COOH.

Figure 2 presents the FT-IR spectrum of modified ShMWCNTs. In Sh-MWCNT-COOH, the peak at $1575 \mathrm{~cm}^{-1}$ is assigned to $\mathrm{C}=\mathrm{C}$ stretching mode of Sh-MWCNT-COOH that forms the framework of CNTs sidewall [16, 17]. The appearance of absorption peaks at $1724(\mathrm{C}=\mathrm{O})$ and 1150 $(\mathrm{C}-\mathrm{O}) \mathrm{cm}^{-1}$ in IR spectra of Sh-MWCNT-COOH clearly 


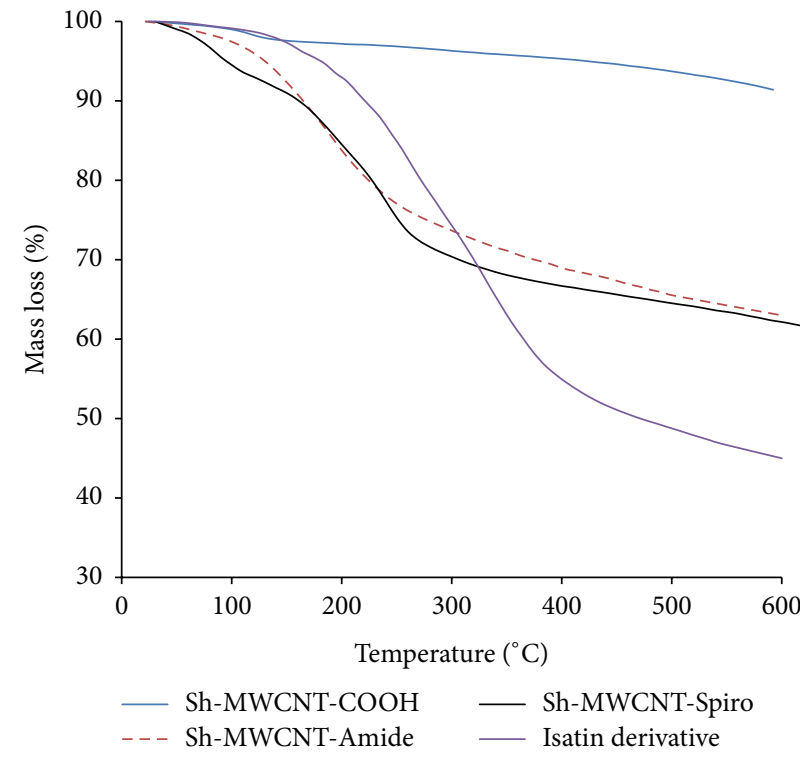

(a)

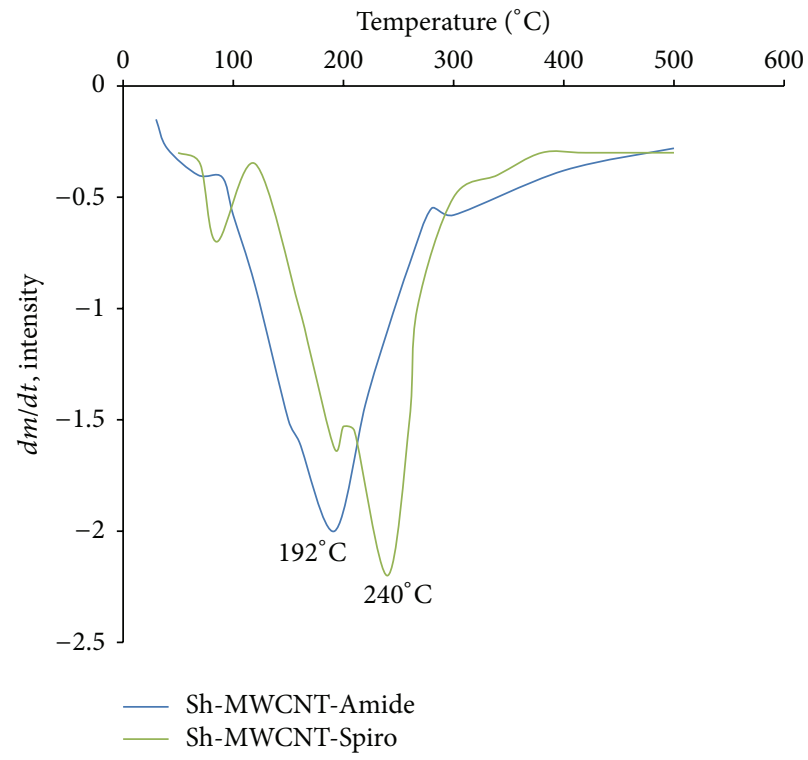

(b)

Figure 3: TGA (a) and DTG (b) curves of modified-MWCNTs in the $\mathrm{N}_{2}\left(10^{\circ} \mathrm{C} \mathrm{min}^{-1}\right)$.

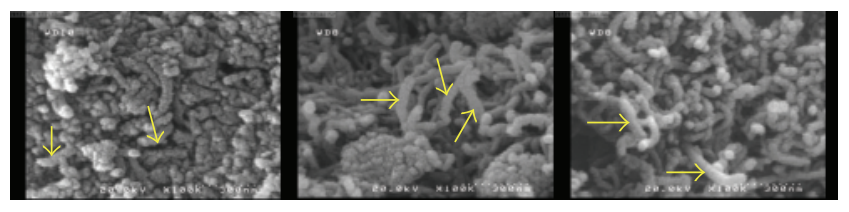

Sh-MWCNT-COOH Sh-MWCNT-Amide Sh-MWCNT-Spiro

FIgURE 4: The FESEM images of modified Sh-MWCNTs. The yellow arrows in Sh-MWCNT-Amide and Sh-MWCNT-Spiro show a slight increase in thickness on the surface of tubes with respect to ShMWCNT-COOH.

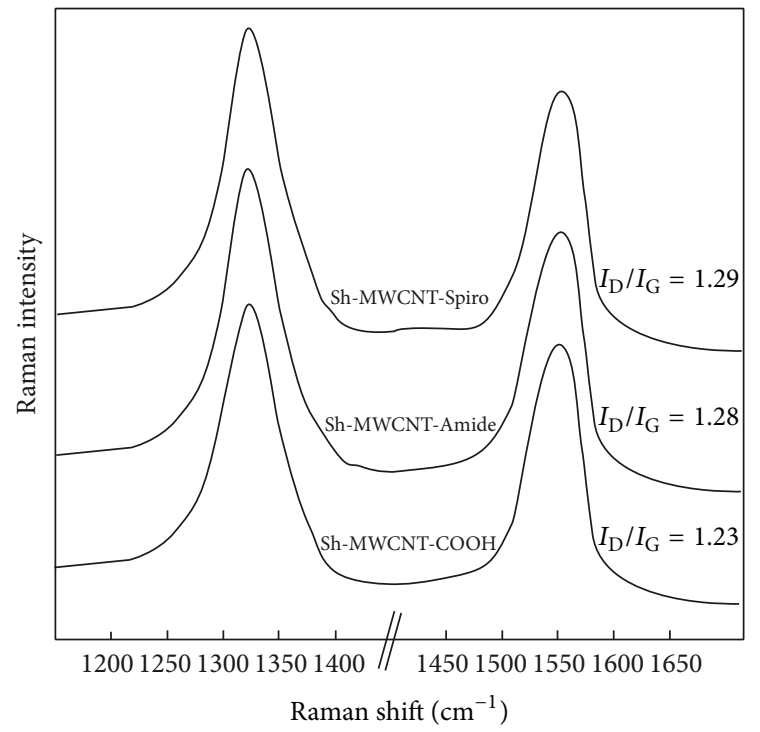

FIgURE 5: The Raman spectra of modified Sh-MWCNTs. The baseline was corrected for the luminescence background.

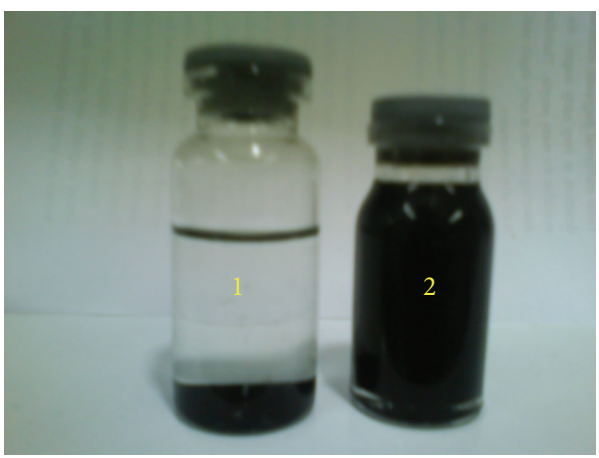

FIGURE 6: The dispersion images of Sh-MWCNT-COOH (1) and ShMWCNT-Spiro (2) in DMF (1 mg/7 mL) after standing for 4 month.

indicates carboxylic groups on the Sh-MWCNTs $[16,17]$. The two bands at around 2800-3000 that can be seen in all spectra can be related to the $\mathrm{C}-\mathrm{H}$ stretching of Sh-MWCNTs defects. In the spectrum of Sh-MWCNT-Amide, the new peaks at $1710,1649,1629$, and $1023 \mathrm{~cm}^{-1}$ can be assigned to the $\mathrm{C}=\mathrm{O}$ of creatinine, amide groups $(\mathrm{C}(=\mathrm{O}) \mathrm{NH}$ linkage $), \mathrm{C}=\mathrm{O}$, and $\mathrm{C}-$ $\mathrm{OH}$ of isatin, respectively, (as compared to $1724 \mathrm{~cm}^{-1}$ for ShMWCNTs- $\mathrm{COOH}$ ), which confirmed the formation of ShMWCNT-Amide. In the spectra of Sh-MWCNT-Spiro, the peaks of $\mathrm{C}-\mathrm{OH}$ isatin and $\mathrm{C}=\mathrm{O}$ creatinine disappear, and the remarkable peak at around $1580-1600 \mathrm{~cm}^{-1}$ appeared which can be assigned to the $\mathrm{C}=\mathrm{N}$ or $\mathrm{C}=\mathrm{C}$ stretching mode (probably overlapped together) of imidazole ring $[17,18,29]$. Also, in the spectra of Sh-MWCNT-Amide and Sh-MWCNT-Spiro the peaks at around 3100-3500, 1450-1600, 1200-1400, and $1000-1180 \mathrm{~cm}^{-1}$ correspond to $\mathrm{N}-\mathrm{H}$ or $\mathrm{OH}$ stretching mode, $\mathrm{C}=\mathrm{C}$ nanotube and aromatic ring modes, and $\mathrm{C}-\mathrm{N}$ and $\mathrm{C}-\mathrm{O}$ stretching modes, respectively. Thus, FT-IR spectra confirm 

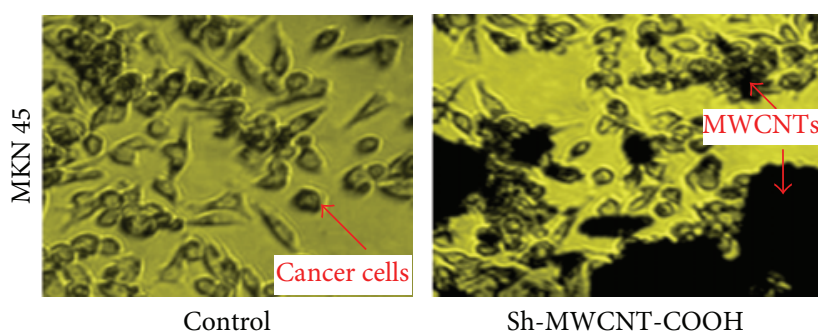

Sh-MWCNT-COOH

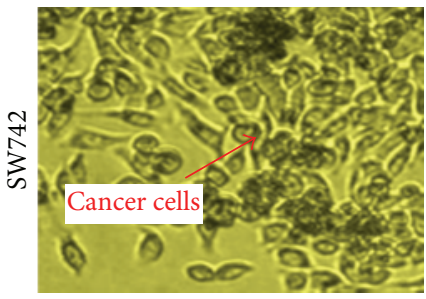

Control

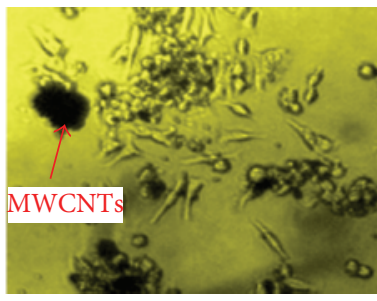

Sh-MWCNT-COOH

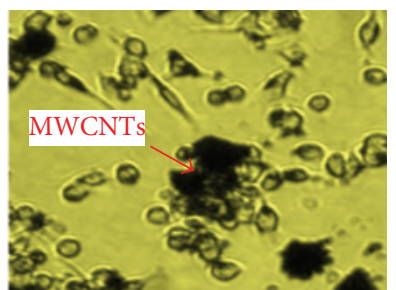

Sh-MWCNT-Amide

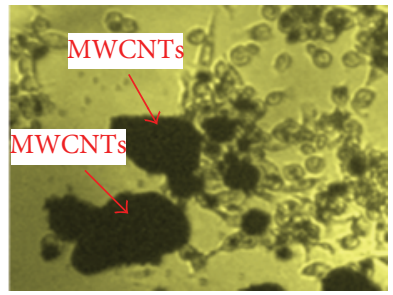

Sh-MWCNT-Amide

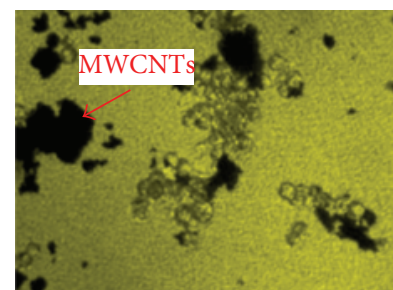

Sh-MWCNT-Spiro

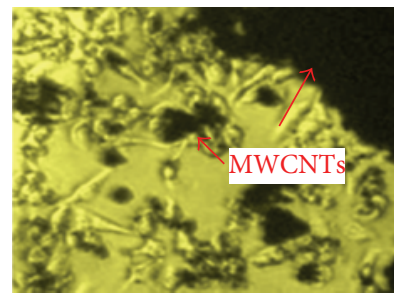

Sh-MWCNT-Spiro

FIGURE 7: The growth of MKN45 and SW742 cancer cells on the samples.

that Sh-MWCNT-COOH has been successfully modified by isatin derivative and Spiro compound.

The best evidence for the functionalization of MWCNTs is the thermogravimetric analysis (TGA, DTG) results, which provide quantitative information on nanotube functionalization. Since Sh-MWCNT-COOH is almost thermally stable, as shown in Figure 3(a), the weight loss before Sh-MWCNTs decomposition can be used to estimate the quantity of the various groups attached to the nanotube. According to Figure 3(a), the TGA graph of Sh-MWCNTAmide exhibits one major decomposition at around 120$320^{\circ} \mathrm{C}$ with weight loss of about $23.41 \%$ that can be assigned to the decomposition of the attached isatin derivative to CNT (as compared with TGA curve of isatin derivative). Also, the major decompositions of Sh-MWCNT-Spiro occurred at around $140-210^{\circ} \mathrm{C}$ and $210-320^{\circ} \mathrm{C}$ with a weight loss of about $8.74 \%$ and $13.62 \%$, respectively, which originate from the decomposition of the residual isatin derivative on the CNT surface and Spiro compound, respectively, (see also DTG curve of Sh-MWCNT-Spiro). On the basis, the fraction of carbon atoms that are functionalized with isatin derivative and Spiro compound in $320^{\circ} \mathrm{C}$ was calculated to be about 1.3 and $0.88 \mathrm{wt} \%$, respectively. The DTG curve provides further evidence for covalent modification of MWCNTs. According to Figure 3(b), the major peak at $192^{\circ} \mathrm{C}$ could be attributed to the loss of the isatin derivative groups bonded to MWCNT. On the other hand, the DTG curve of ShMWCNT-Spiro shows two peaks at 192 and $240^{\circ} \mathrm{C}$ which can be assigned to the loss residual isatin derivative and Spiro compound, respectively. Overall, these results successfully confirm the functionalization of Sh-MWCNT-COOH with these compounds.

More evidence for the functionalization of Sh-MWCNTs was obtained by FE-SEM images. In Figure 4, FE-SEM images of modified Sh-MWCNTs are shown. In the SEM image of Sh-MWCNT-COOH, it seems that the uniform surfaces of nanotubes are relatively smooth. On the other hand, for the Sh-MWCNT-Amide and Sh-MWCNT-Spiro, a tubular layer of uniform organic compounds (isatin derivative and Spiro compound) is clearly present on the surface of the MWCNT (the rough part), and their diameters are slightly increased as compared to that of Sh-MWCNT-COOH. It may be due to covalently bonded isatin derivative and Spiro compound on the surface of the MWCNT as compared to that of Sh-MWCNT-COOH. These structures, Sh-MWCNTAmide and Sh-MWCNT-Spiro, are quite different from those of the starting Sh MWCNT-COOH, as depicted in Figure 4.

The Raman spectra of functionalized Sh-MWCNTs (Figure 5) showed a slight increase of the D-band at $\sim 1330 \mathrm{~cm}^{-1}$, which is attributed to the defects and disorderinduced peaks, relative to the G-band $\left(\sim 1560 \mathrm{~cm}^{-1}\right)$, which originates from the in-plane tangential stretching mode of carbon-carbon bonds [30], $\left(I_{\mathrm{D}} / I_{\mathrm{G}}=1.28-1.29\right)$ compared to Sh-MWCNT-COOH $\left(I_{\mathrm{D}} / I_{\mathrm{G}}=1.23\right)$. These results (approximately equal $1.23 \approx 1.28-1.29$ ) indicate which reactions do not affect the graphite structure of Sh-MWCNT-COOH [16-18].

A fair idea whether the modification on the carbon nanotubes has been achieved or not is the dispersion test. Figure 6 presents a photograph of the dispersion of Sh-MWCNT$\mathrm{COOH}$ and Sh-MWCNT-Spiro in DMF. As it can be seen from Figure 6, Sh-MWCNT-COOH is insoluble in DMF, while the Sh-MWCNT-Spiro can be directly dispersed in DMF homogeneously and no precipitation was found even after it was sealed for 4 months at room temperature. These results indicate which Sh-MWCNT-COOH was functionalized by Spiro compound.

The effect of functionalized Sh-MWCNTs on toxicity of gastric and colon cancer cell lines was investigated. The used biological data in this study revealed anticancer activity against MKN-45 and SW742. Figure 7 shows images of cell culture on the modified Sh-MWCNTs and control. Also, it shows targeting cancerous cells by CNTs in both gastric and colon cells. The images of control samples related to the culture of cancerous cells on the tissue culture polystyrene (TCPS) surface show the cells of well adhesion and proliferation on the TCPS surface. Figures of modified 


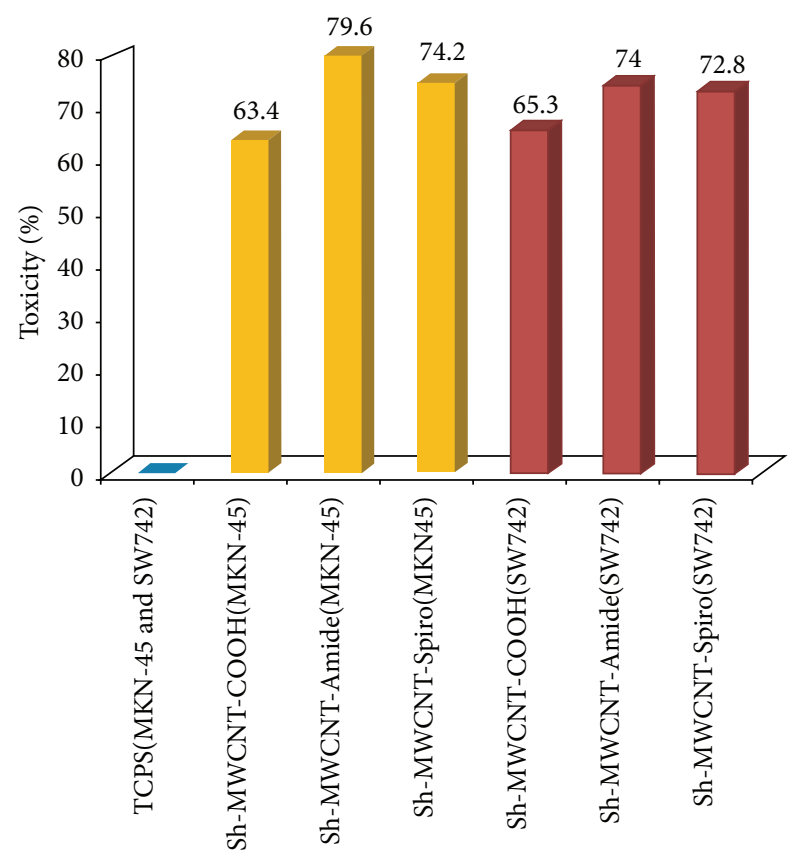

FIGURE 8: MTT assay for TCPS and modified MWCNTs after $48 \mathrm{hr}$.

Sh-MWCNTs show the effect of the gastric and colon cancerous cells in the vicinity of the modified Sh-MWCNTs and the control sample. These show that the modified Sh-MWCNTs will diffuse into and on cancerous cells.

Figure 8 shows an MTT assay for TCPS (control), ShMWCNT-COOH, Sh-MWCNT-Amide, and Sh-MWCNTSpiro on MKN-45 and SW742. The results showed high toxicity of modified MWCNTs for MKN-45 (about 63\%-80\%) compared to SW742 (about 65\%-74\%). Also, it indicates which toxicity of Sh-MWCNT-Amide in MKN-45 (79.6\%) and SW742 (74\%) is more than the other samples. We dictate which concentration of the samples could be considered similar $(100 \mu \mathrm{g} / \mathrm{mL})$. Overall, these results demonstrated that modified Sh-MWCNTs are a powerful agent for both MKN45 and SW742, in which it leads to kill cancer cells about 72$80 \%$.

So, functionalization of these groups is very effective for the toxicity increase of MWCNTs that can be used to conjugate with drugs of anticancer or biochemically materials.

\section{Conclusion}

We have firstly demonstrated the anticancer agents by functionalization of Sh-MWCNTs. We have designed and synthesized novel derivative of isatin, which had potential use for cellular toxicity especially cancerous cells. The reported results demonstrated the functionalization of Sh-MWCNT$\mathrm{COOH}$ by isatin and Spiro derivatives. Also, our results demonstrated the high toxicity of modified Sh-MWCNTs on both gastric and colon cancer cells (72-80\%).

\section{Conflict of Interests}

The authors report no conflict of interests in this work.

\section{Acknowledgment}

The authors are grateful for the financial and encouragement support provided by Research vice Presidency of Ayatollah Amoli Branch, Islamic Azad University and Iranian Nanotechnology Initiative (Government of Iran).

\section{References}

[1] S. Reich, C. Thomsen, and J. Maultzsch, Carbon Nanotubes, Wiley-VCH, Berlin, Germany, 2010.

[2] H. Hu, Y. Ni, V. Montana, R. C. Haddon, and V. Parpura, "Chemically functionalized carbon nanotubes as substrates for neuronal growth," Nano Letters, vol. 4, no. 3, pp. 507-511, 2004.

[3] N. Venkatesan, J. Yoshimitsu, Y. Ito, N. Shibata, and K. Takada, "Liquid filled nanoparticles as a drug delivery tool for protein therapeutics," Biomaterials, vol. 26, no. 34, pp. 7154-7163, 2005.

[4] T. S. Koblenz, J. Wassenaar, and J. N. H. Reek, "Reactivity within a confined self-assembled nanospace," Chemical Society Reviews, vol. 37, no. 2, pp. 247-262, 2008.

[5] Y. Baudry, G. Bollot, V. Gorteau et al., "Molecular recognition by synthetic multifunctional pores in practice: are structural studies really helpful?" Advanced Functional Materials, vol. 16, no. 2, pp. 169-179, 2006.

[6] C. J. Gannon, P. Cherukuri, B. I. Yakobson et al., "Carbon nanotube-enhanced thermal destruction of cancer cells in a noninvasive radiofrequency field," Cancer, vol. 110, no. 12, pp. 2654-2665, 2007.

[7] Z. Liu, X. Sun, N. Nakayama-Ratchford, and H. Dai, "Supramolecular chemistry on water- soluble carbon nanotubes for drug loading and delivery," ACS Nano, vol. 1, no. 1, pp. 50-56, 2007.

[8] P. L. McEuen, M. S. Fuhrer, and H. Park, "Single-walled carbon nanotube electronics," IEEE Transactions on Nanotechnology, vol. 1, no. 1, pp. 78-85, 2002.

[9] T. Dürkop, S. A. Getty, E. Cobas, and M. S. Fuhrer, "Extraordinary mobility in semiconducting carbon nanotubes," Nano Letters, vol. 4, no. 1, pp. 35-39, 2004.

[10] A. A. Shvedova, E. R. Kisin, D. Porter et al., "Mechanisms of pulmonary toxicity and medical applications of carbon nanotubes: two faces of Janus?" Pharmacology and Therapeutics, vol. 121, no. 2, pp. 192-204, 2009.

[11] J. E. Podesta, K. T. Al-Jamal, M. A. Herrero et al., "Antitumor activity and prolonged survival by carbon-nanotube-mediated therapeutic sirna silencing in a human lung xenograft model," Small, vol. 5, no. 10, pp. 1176-1185, 2009.

[12] J. Chen, S. Chen, X. Zhao, L. V. Kuznetsova, S. S. Wong, and I. Ojima, "Functionalized single-walled carbon nanotubes as rationally designed vehicles for tumor-targeted drug delivery," Journal of the American Chemical Society, vol. 130, no. 49, pp. 16778-16785, 2008.

[13] M. Popławska, G. Z. Zukowska, S. Cudziło, and M. Bystrzejewski, "Chemical functionalization of carbon-encapsulated magnetic nanoparticles by 1,3-dipolar cycloaddition of nitrile oxide," Carbon, vol. 48, no. 4, pp. 1318-1320, 2010. 
[14] F. Cataldo, "A study on the action of ozone on multiwall carbon nanotubes," Fullerenes Nanotubes and Carbon Nanostructures, vol. 16, no. 1, pp. 1-17, 2008.

[15] F. Borondics, M. Bokor, P. Matus, K. Tompa, S. Pekker, and E. Jakab, "Reductive functionalization of carbon nanotubes," Fullerenes Nanotubes and Carbon Nanostructures, vol. 13, no. 1, pp. 375-382, 2005.

[16] H. Tahermansouri, M. Azadfar, and S. Heidari, "Functionalization and toxicity effect of multi-walled carbon nanotubes with urea derivatives via microwave irradiation," Fullerenes, Nanotubes, and Carbon Nanostructures, vol. 21, no. 7, pp. 568578, 2013.

[17] H. Tahermansouri, Y. Aryanfar, and E. Biazar, "Synthesis, characterization, and the influence of functionalized multi-walled carbon nanotubes with creatinine and 2-aminobenzophenone on the gastric cancer cells," Bulletin- Korean Chemical Society, vol. 34, pp. 149-153, 2013.

[18] J. Azizian, H. Tahermansouri, D. C. Khoei, K. Yadollahzadeh, and A. S. Delbari, "Microwave-induced chemical functionalization of carboxylated multi-walled nanotubes with 2,3diaminopyridine," Fullerenes Nanotubes and Carbon Nanostructures, vol. 20, no. 2, pp. 183-190, 2012.

[19] J. Azizian, D. C. Khoei, H. Tahermansouri, and K. Yadollahzadeh, "Functionalization of carboxylated multi-walled carbon nanotubes with 1, 4-phenylendiamine, phenylisocyanate and phenylisothiocyanate," Fullerenes Nanotubes and Carbon Nanostructures, vol. 19, no. 8, pp. 753-760, 2011.

[20] J. Azizian, H. Tahermansouri, E. Biazar, S. Heidari, and D. C. Khoei, "Functionalization of carboxylated multiwall nanotubes with imidazole derivatives and their toxicity investigations," International Journal of Nanomedicine, vol. 5, no. 1, pp. 907-914, 2010.

[21] H. Tahermansouri, M. Atghaee, and M. Azadfar, "The chemical functionalization of multi-wall nanotubes with Methyl 2(2-amino-4-oxothiazol-5(4H)-ylidene) Acetate and Phenylhydrazine," Oriental Journal of Chemistry, vol. 27, no. 4, pp. 13251329, 2011

[22] O. Moradi, M. Yari, K. Zare, B. Mirza, and F. Najafi, "Carbon nanotubes: a review of chemistry principles and reactions," Fullerenes Nanotubes and Carbon Nanostructures, vol. 20, no. 2, pp. 138-151, 2012.

[23] A. Aqel, K. M. M. A. El-Nour, R. A. A. Ammar, and A. AlWarthan, "Carbon nanotubes, science and technology part (I) structure, synthesis and characterisation," Arabian Journal of Chemistry, vol. 5, no. 1, pp. 1-23, 2012.

[24] D. Cui, F. Tian, C. S. Ozkan, M. Wang, and H. Gao, "Effect of single wall carbon nanotubes on human HEK293 cells," Toxicology Letters, vol. 155, no. 1, pp. 73-85, 2005.

[25] J. M. Wörle-Knirsch, K. Pulskamp, and H. F. Krug, "Oops they did it again! Carbon nanotubes hoax scientists in viability assays," Nano Letters, vol. 6, no. 6, pp. 1261-1268, 2006.

[26] H.-T. Chou, T.-P. Wang, C.-Y. Lee, N.-H. Tai, and H.-Y. Chang, "Photothermal effects of multi-walled carbon nanotubes on the viability of BT-474 cancer cells," Science and Engineering $C$, vol. 33, pp. 989-995, 2013.

[27] X. Liu, R. H. Hurt, and A. B. Kane, "Biodurability of singlewalled carbon nanotubes depends on surface functionalization," Carbon, vol. 48, no. 7, pp. 1961-1969, 2010.

[28] N. R. Penthala, T. R. Yerramreddy, N. R. Madadi, and P. A. Crooks, "Synthesis and in vitro evaluation of N-alkyl-3hydroxy-3-(2-imino-3-methyl- 5-oxoimidazolidin-4-yl)indo- lin-2-one analogs as potential anticancer agents," Bioorganic and Medicinal Chemistry Letters, vol. 20, no. 15, pp. 4468-4471, 2010.

[29] Z. Yang, H. Pu, and J. Yin, "Covalent functionalization of multiwalled carbon nanotubes by polyvinylimidazole," Materials Letters, vol. 59, no. 22, pp. 2838-2841, 2005.

[30] H. Hiura, T. W. Ebbesen, K. Tanigaki, and H. Takahashi, "Raman studies of carbon nanotubes," Chemical Physics Letters, vol. 202, no. 6, pp. 509-512, 1993. 

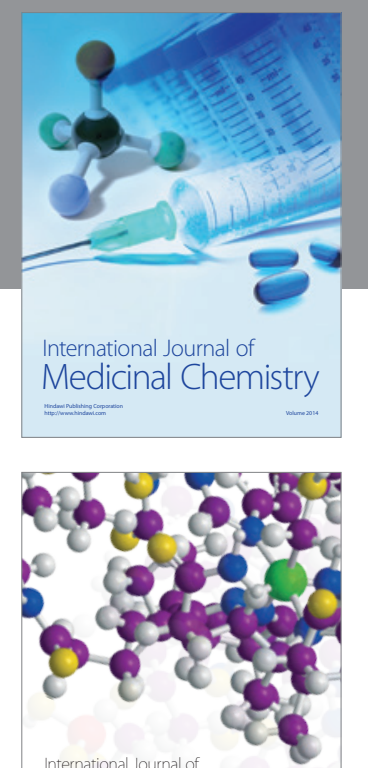

\section{Carbohydrate} Chemistry

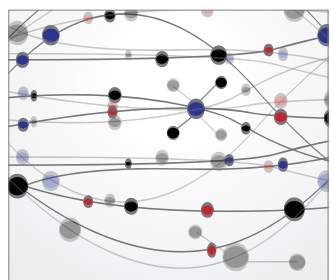

The Scientific World Journal
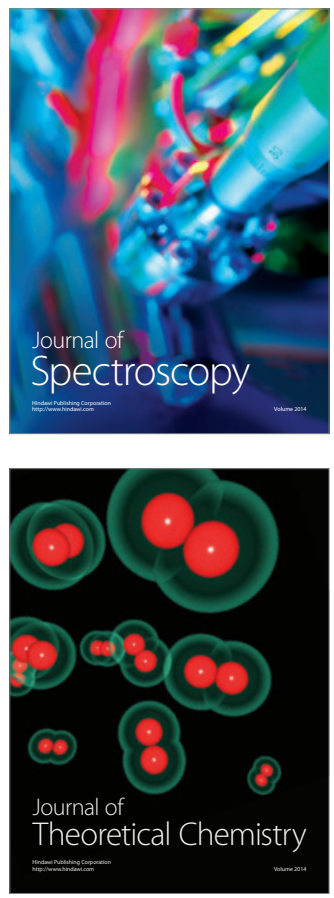
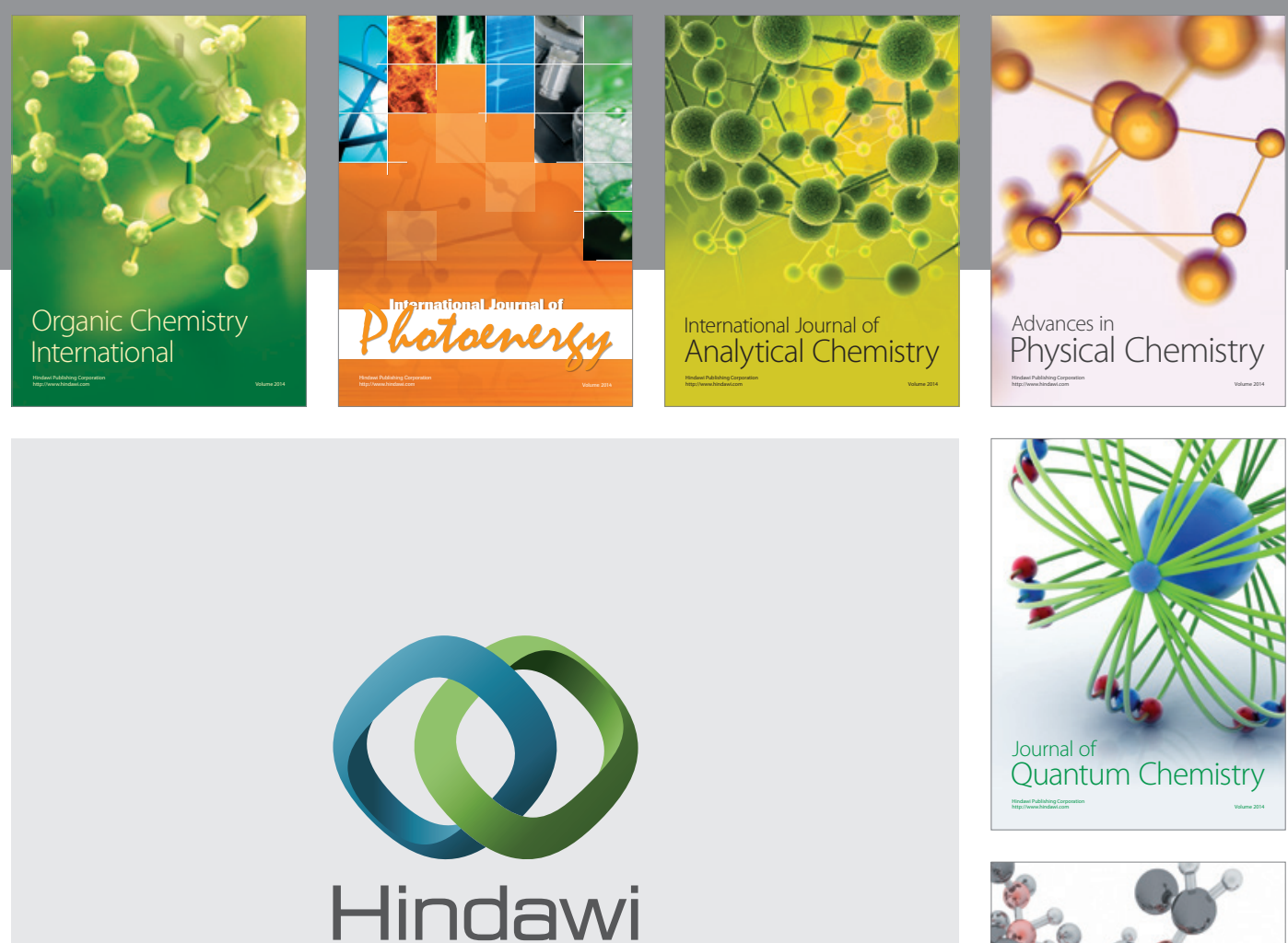

Submit your manuscripts at

http://www.hindawi.com

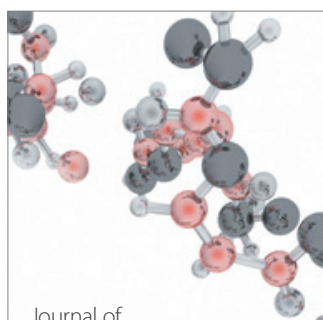

Analytical Methods

in Chemistry

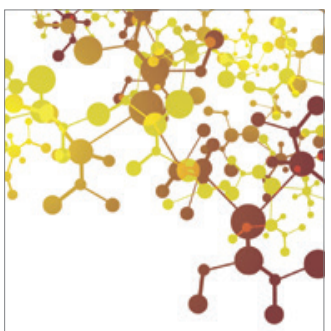

Journal of

Applied Chemistry

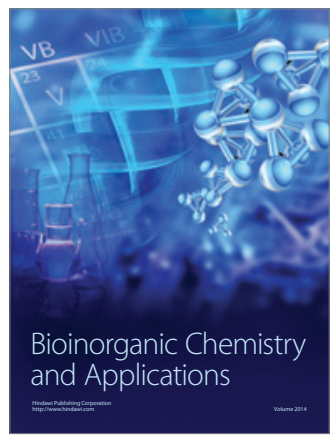

Inorganic Chemistry
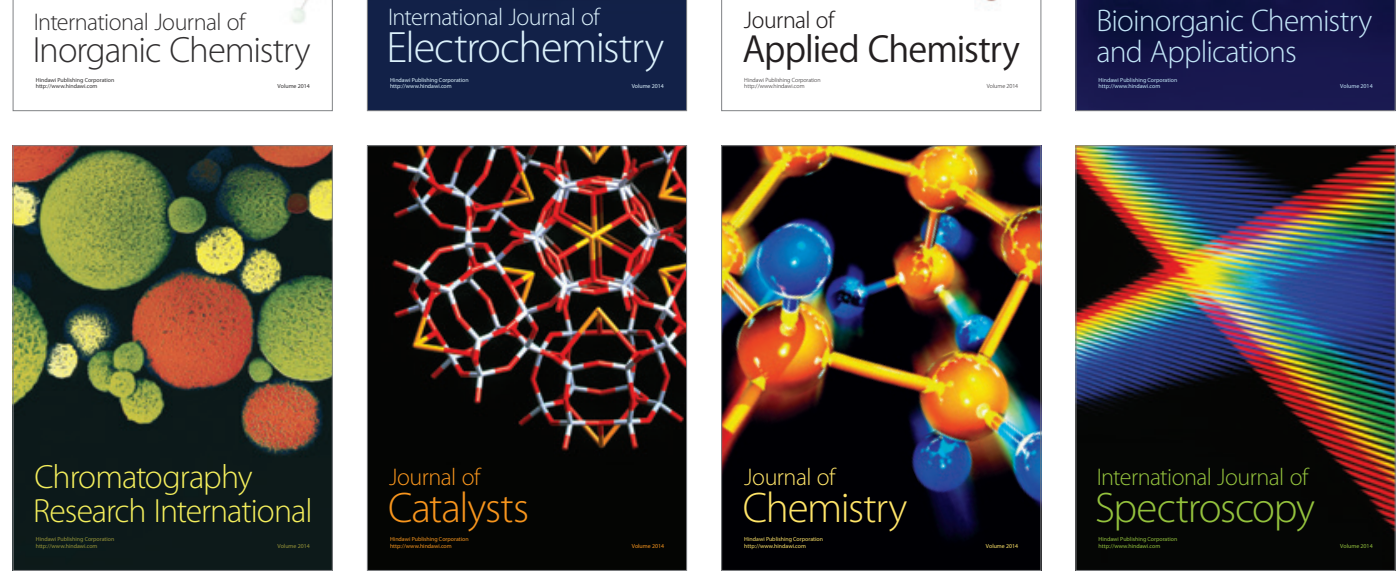